\title{
Study of conjugate heat transfer accompanying mixed convection in a vertical tube submitted to a step of entry temperature
}

\author{
O. Kholai ${ }^{1,2}$, M. Kadja ${ }^{2}$ \& T. H. Mai ${ }^{1}$ \\ ${ }^{1}$ Laboratoire de Thermomécanique, Faculté des sciences, Reims, France \\ ${ }^{2}$ Département de Génie Mécanique, Université de Constantine, \\ Constantine, Algeria
}

\begin{abstract}
The paper presents the results of a numerical study on transient mixed laminar convection for the case of an ascending flow inside a vertical tube in which the wall conduction is significant. The outer surface of the tube is submitted to a convective heat exchange. The transient regime is provoked by a step type perturbation in temperature at entry of the tube. The governing equations are solved using a finite volume method. Results are presented for water $(\mathrm{Pr}=5)$ and for two values of Grashof numbers of $10^{4}$ and $10^{5}$. This method shows that no matter what the value of the imposed step is, it provokes the birth of a big recirculation cell next to the wall, and gives rise to an instability situated between the cool and the hot regions of the flow.
\end{abstract}

Keywords: transient convection, temperature step, convective heat exchange, finite volume method, instabilities.

\section{Introduction}

Conjugate heat transfer (mixed convection + conduction) in transient regime in internal flows is present in many industrial installations: such as compact heat exchangers, solar collectors, cooling of electronic components etc. As a matter of fact, the importance and complexity of this phenomenon and the consequences have stimulated the interest of many researchers: so that a very important number of studies have been done in this field.

Nguyen et al. [1] performed a numerical study on transient mixed convection in vertical tubes having a wall, which is submitted to variable heat flux. Their 
results indicate that the increase in heat flux gives rise to a recirculation zone, which increases in intensity and size as a function of time. Experimentally Morton et al. [2] have confirmed the existence of a recirculation cell near the tube axis. For conjugate heat transfer problems (conduction in the wall and convection in the fluid, inside a tube), Bilir and Ates [3] performed a parametric study in a vertical tube submitted to convective exchange at the outer surface. Bernier and Baliga $[4,5]$ studied the problem of mixed convection by taking into account wall conduction for an ascending flow where the tube is submitted to a uniform heat flux. They have shown that axial diffusion of heat through the tube wall becomes very big for high conductivity and thickness of the wall and for a low Peclet number. Recirculation zones and instability have been shown to occur by Zgal et al. [6] and Su and Chung [7]. More recently Mai et al. [8], Popa and Mai [9] and Mai et al. [10] have focussed attention on a more complex problem of heat transfer instability. They have shown that a perturbation of mixed convection in a vertical tube via a step rise in temperature (of mass flow rate) at entry, gives rise to instabilities of thermo-hydraulic flow structure and to the creation of recirculation cells inside the fluid.

The present study consists in analysing the phenomenon of transient instability of a flow in mixed laminar convection in a circular vertical duct when the entry and the outer surface of the duct are submitted to a step change in temperature (positive or negative) and to a convective exchange with ambient air, respectively. The influence of these two conditions and also the ratio of thermal diffusivities (wall/fluid) on the transient behaviour of the flow are studied in detail.

\section{Problem formulation}

The considered problem consists in studying conjugate heat transfer for a laminar flow inside a vertical tube submitted to convective exchange with air through a section of the outer wall. The wall thickness is taken into consideration and is equal to $\delta=0.1 \mathrm{D}$. Adiabatic sections have been added upwind and downwind of the cooled section so as to permit the study of longitudinal thermal diffusion in the fluid and the wall. The configuration and the coordinate system of the tube are given in Fig. 1. The governing equations of this problem are: the continuity equation, the Navier-Stokes equations and the equation of energy. The study is based on the following simplifying assumptions:

-The fluid is Newtonian and incompressible

-The thermo-physical properties of the fluid and the solid are constant

-The fluid obeys the Boussinesq approximation: density is constant except in the natural convection generating terms (density varies linearly with temperature)

-The flow is laminar and in transient regime

-Viscous dissipation and radiation heat transfer are negligible.

By taking these assumptions into account and adopting the following dimensionless variables: 


$$
\begin{gathered}
\mathrm{R}=\frac{\mathrm{r}}{\mathrm{D}}, \quad \mathrm{Z}=\frac{\mathrm{z}}{\mathrm{D}}, \quad \mathrm{U}=\frac{\mathrm{u}}{\mathrm{U}_{\mathrm{av}}}, \quad \mathrm{V}=\frac{\mathrm{v}}{\mathrm{U}_{\text {av }}}, \quad \theta=\frac{\mathrm{T}-\mathrm{T}_{0}}{\mathrm{~T}_{\text {in }}-\mathrm{T}_{0}} \\
\mathrm{P}=\frac{\mathrm{p}+\rho_{0} \mathrm{gz}}{\rho_{0} \mathrm{U}_{\mathrm{av}}^{2}}, \quad \tau=\left(\frac{\mathrm{t}}{\mathrm{D} / \mathrm{U}_{\text {av }}}\right), \quad \text { and } \quad \alpha^{*}=\frac{\alpha}{\alpha_{\mathrm{f}}}
\end{gathered}
$$

where $\mathrm{u}$ and $\mathrm{v}$ are the velocity components in the $\mathrm{z}$ and $\mathrm{r}$ directions respectively, $\mathrm{T}$ is the temperature, $\mathrm{p}$ is the pressure, $\mathrm{D}$ is the diameter of the tube, $\mathrm{t}$ is the time, $\mathrm{U}_{\mathrm{av}}$ is the mean axial velocity at inlet. $\mathrm{g}$ is the acceleration due to gravity. $\mathrm{T}_{0}$ is the ambient temperature. $\rho_{0}$ is the fluid density at $T_{0}$. The index 'in' stands for inlet and ' $\mathrm{f}$ ' for fluid.

The governing equations can be written in the following form in cylindrical coordinates:

$$
\begin{gathered}
\frac{\partial \mathrm{U}}{\partial \mathrm{Z}}+\frac{1}{\mathrm{R}}\left(\frac{\partial \mathrm{RV}}{\partial \mathrm{R}}\right)=0 \\
\frac{\partial \mathrm{U}}{\partial \tau}+\mathrm{U} \frac{\partial \mathrm{U}}{\partial \mathrm{Z}}+\mathrm{V} \frac{\partial \mathrm{U}}{\partial \mathrm{R}}=-\frac{\partial \mathrm{P}}{\partial \mathrm{Z}}+\frac{1}{\mathrm{Re}}\left[\frac{\partial^{2} \mathrm{U}}{\partial \mathrm{Z}^{2}}+\frac{1}{\mathrm{R}} \frac{\partial}{\partial \mathrm{R}}\left(\mathrm{R} \frac{\partial \mathrm{U}}{\partial \mathrm{R}}\right)\right]+\frac{\mathrm{Gr}}{\mathrm{Re}^{2}} \theta \\
\frac{\partial \mathrm{V}}{\partial \tau}+\mathrm{U} \frac{\partial \mathrm{V}}{\partial \mathrm{Z}}+\mathrm{V} \frac{\partial \mathrm{V}}{\partial \mathrm{R}}=-\frac{\partial \mathrm{P}}{\partial \mathrm{R}}+\frac{1}{\mathrm{Re}}\left[\frac{\partial^{2} \mathrm{~V}}{\partial \mathrm{Z}^{2}}+\frac{1}{\mathrm{R}} \frac{\partial}{\partial \mathrm{R}}\left(\mathrm{R} \frac{\partial \mathrm{V}}{\partial \mathrm{R}}\right)-\frac{\mathrm{V}}{\mathrm{R}^{2}}\right] \\
\frac{\partial \theta}{\partial \tau}+\mathrm{U} \frac{\partial \theta}{\partial \mathrm{Z}}+\mathrm{V} \frac{\partial \theta}{\partial \mathrm{R}}=\frac{\alpha^{*}}{\operatorname{RePr}}\left[\frac{\partial^{2} \theta}{\partial \mathrm{Z}^{2}}+\frac{1}{\mathrm{R}} \frac{\partial}{\partial \mathrm{R}}\left(\mathrm{R} \frac{\partial \theta}{\partial \mathrm{R}}\right)\right]
\end{gathered}
$$

where $\mathrm{U}, \mathrm{V}, \mathrm{P}, \theta$ are the adimensional variables (adimensional velocity components, adimensional pressure and adimensional temperature), $\mathrm{R}$ and $\mathrm{Z}$ are the adimensional coordinates and $\mathrm{Re}, \mathrm{Pr}, \mathrm{Gr}$ and $\alpha^{*}$ are the dimensionless parameters controlling the problem:

$$
\begin{array}{ll}
\operatorname{Re}=\frac{\mathrm{U}_{\mathrm{av}} \mathrm{D}}{v} & \text { Reynolds number } \\
\operatorname{Pr}=\frac{v}{\alpha} & \text { Prandtl number } \\
\mathrm{Gr}=\frac{\mathrm{g} \beta \Delta \mathrm{TD}^{3}}{v^{2}} & \text { Grashof number based on the heat flux } \\
\alpha^{*}=\frac{\alpha}{\alpha_{\mathrm{f}}} & \text { Ratio of thermal diffusivities solid/fluid }
\end{array}
$$

where $\beta$ is the thermal expansion coefficient and $v$ is the kinematic viscosity of the fluid.

Initially (i.e. at $\tau=0$ ), the common boundary conditions are based on a parabolic profile of velocity and a uniform temperature profile at entry, a symmetry of the hydrodynamic and temperature fields with respect to the tube axis and a completely developed profile at exit. 
On the other hand the thermal condition on the wall $(\mathrm{r}=\mathrm{D} / 2+\delta)$ is:

If $\mathrm{Z}<\mathrm{L}_{1}$ then $\frac{\partial \theta}{\partial \mathrm{R}}=0 \quad$ (adiabatic condition)

If $\quad \mathrm{L}_{1} \leq \mathrm{Z} \leq \mathrm{L}_{2}$ then $\left.\frac{\partial \theta}{\partial \mathrm{R}}\right|_{\mathrm{w}}=-\frac{\mathrm{hD}}{\lambda} \theta_{\mathrm{w}} \quad$ (convective exchange condition)

If $\quad \mathrm{Z}>\left(\mathrm{L}_{1}+\mathrm{L}_{2}\right)$ then $\frac{\partial \theta}{\partial \mathrm{R}}=0 \quad$ (adiabatic condition)

where $\mathrm{h}$ is the convective heat transfer coefficient and $\lambda$ the thermal conductivity of the tube.

Using the above boundary conditions, one can obtain the developed (permanent) regime after a time $\left(\tau=\tau_{\mathrm{e}}\right)$. One then perturbs the flow using a step change in inlet temperature, such as:

At $\tau=\tau_{\mathrm{e}}$ and $\mathrm{Z}=0, \theta=\theta \pm \Delta \theta$

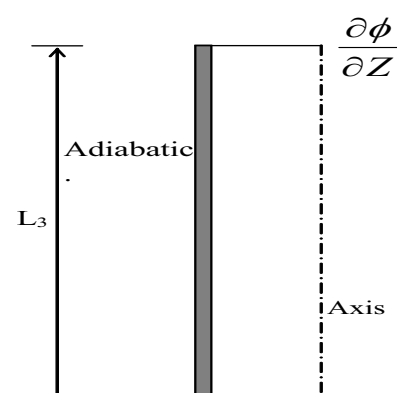

Figure 1: Geometric configuration of the studied case.

\section{Numerical procedure}

The equations presented above have been integrated and discretized on a staggered grid following the finite volume approach explained in [11]. We have chosen the SIMPLE algorithm in order to solve for pressure. The power law-differencing scheme has been employed to calculate the convective fluxes in the transport equations for momentum and energy . Temporal discretization has been achieved using an implicit unconditionally stable scheme. The physical domain (solid + fluid) has been treated as a heterogeneous medium, the kinematic viscosity being supposed equal to infinity in the solid region. At the interface, the thermal diffusivity was evaluated using the harmonic mean between that of the fluid and that of the solid [11]. The resulting algebraic equations have been solved using the iterative line-by-line method associated with the tri-diagonal matrix algorithm (TDMA). Convergence is considered to be reached when the maximum relative change in velocities and temperatures 
during two consecutive time increments is less than $10^{-4}$. On the basis of many tests, the mesh adopted is non-uniform and consists of 102 nodes in the axial direction and 38 nodes in the radial direction, from which 31 nodes are in the fluid and 7 nodes in the solid wall.

\section{Results and discussions}

In this study, the results obtained correspond to the mixed convection of water $(\mathrm{Pr}=5)$ in a vertical tube submitted on one hand to a convective exchange and on the other hand perturbed at entry via a step change in temperature (positive or negative). Many numerical simulations have been done. They concern the influence of the step change in temperature at entry on the stability of the thermal and hydrodynamic fields for two different Grashof numbers: $10^{4}$ and $10^{5}$, and also the influence of the thermal diffusivity ratio (wall/fluid) on the temperature of the interface.

\subsection{Validation}

The results of the model used in the present study have been compared with the corresponding analytic results obtained by Kakac and Yener [12]. Fig. 2 illustrates the axial evolution of the Nusselt number $\mathrm{Nu}_{\mathrm{z}}$ for the case of forced convection $(\mathrm{Gr}=0)$ in a tube submitted to a uniform heat flux. One can notice a very good accord between the model and the analytic solution.

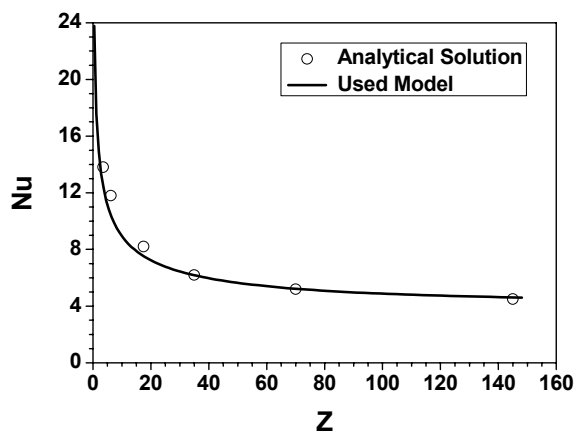

Figure 2: Validation of the model for an average Nusselt number (forced convection).

\subsection{Effect of the thermal diffusivities ratio}

The effect of the thermal diffusivity ratio $\alpha^{*}$ (wall/fluid) on the axial evolution of the interfacial temperature is presented in Fig. 3. One can notice that the increase of this ratio improves the heat transfer by conduction through the wall, which causes a remarkable decrease of the temperature at the interface. The value of the latter tends to that of the ambient temperature when the ratio becomes equal to infinity. 


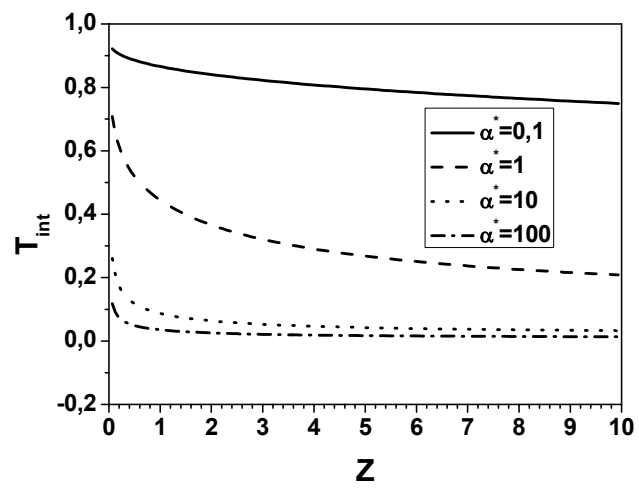

Figure 3: Effect of the ratio of thermal diffusivities on the temperature at the interface.

\subsection{Structure of the thermal and flow fields}

Fig. 4(a) shows the variation of the dynamic and thermal fields as a function of the increase in the temperature step for a given time $(\tau=200)$ and a Grashof number of $10^{4}$. One can notice the presence of a recirculation zone stuck on the wall. This zone is due to the increase in ascending forces caused by natural convection after having perturbed the flow with a positive temperature step at entry. The appearance of this zone is due to the fact that the flow is accelerated in the central region of the tube and decelerated next to the wall. In effect, due to reheating, a density differential establishes itself inside the fluid, the density of the fluid situated in the central zone diminishes with respect to that near to the wall (Boussinesq hypothesis). As the temperature step increases, the recirculation cell intensifies and its size becomes bigger and ends by occupying the totality of the tube.

In Fig. 4(b), corresponding to the thermal field, one can notice a stratification of the isotherms. They are thinner near to the entry of the tube and become larger and larger as one approaches the fluid exit. On the wall one can notice that the radial temperature gradient is negligible due to the importance of the thermal diffusivity of the material with respect to that of the fluid. On the other hand one can clearly see a significant axial temperature gradient, as shown by the isotherms, which are parallel. The increase of the temperature step causes a more condensed stratification and a high heat transfer between the middle of the tube and the wall.

The transient behaviour of the flow is shown in Figs. 5 and 6 for the case of a positive step of 0.6 (reheating) or a negative step of -0.6 (cooling). If a negative step is imposed at entry, one notices that the intensity of the recirculation zone becomes weaker as time increases. This causes a decrease of the important fluid current at the centre of the tube. The dynamic instability equally entrains a 
thermal disequilibrium inside the flow. This is due to the interaction between the fluid cooled next to the wall and the fluid heated next to the tube axis. This instability is clearly remarked for the times $\tau=12,19,29$ and 39 .
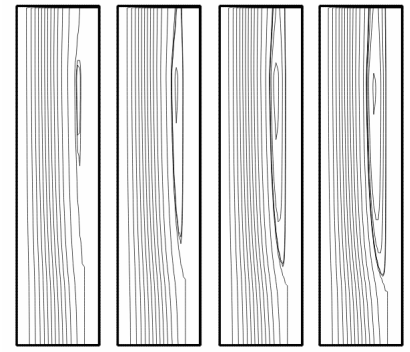

(a)

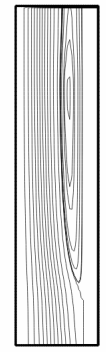

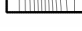
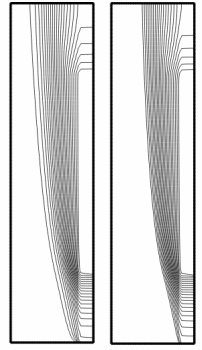
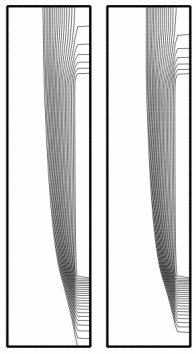

(b)

Figure 4: Effect of the temperature step on the streamfunction and the isotherms, $\mathrm{Gr}=10^{4}, \Delta \theta=0.2,0.4,0.6,0.8,1$.
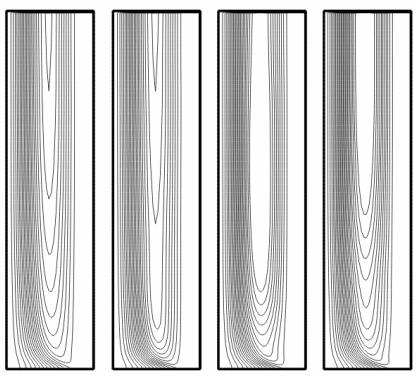

(a)
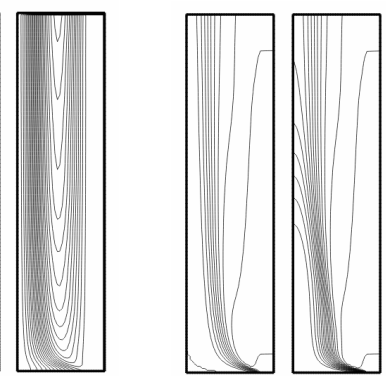

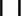

(b)

Figure 5: Evolution of the dynamic field (a) and thermal field (b) as a function of time $\tau=0,12,19,29,39$ and 130 for $\mathrm{Gr}=10^{5}$ and $\Delta \theta=-0.6$.

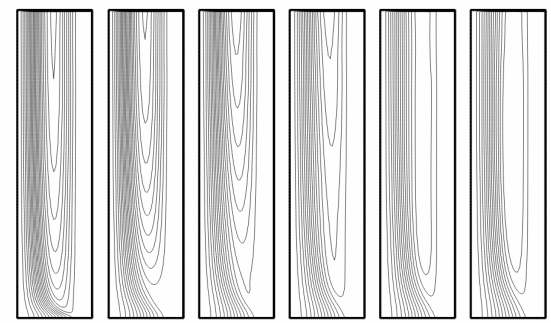

(a)
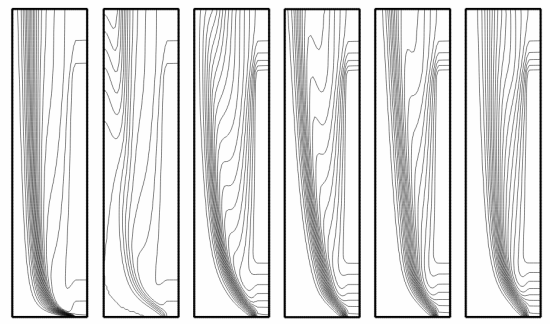

(b)

Figure 6: Evolution of the dynamic field (a) and thermal field (b) as a function of time $\tau=0,8,16,24$, and 39 for $\mathrm{Gr}=10^{5}$ and $\Delta \theta=0.6$. 
After a sudden perturbation of the flow via a positive step (reheating) of fig. 6 , the fluid undergoes a local acceleration at entry and especially near the axis of symmetry, consisting of the reverse current next to the tube wall so as to obey the law of conservation of mass. This can be explained by the fact that convective exchange applied to the outer surface cools the entire region neighbouring the wall. On the other hand, the central region of the tube is heated due to the temperature step. The increase of time $\tau=0,8,16,24$ and 39 shows that the region touched by the instability is situated between the cooled region and the heated one inside the fluid.

\section{Conclusion}

In this paper a numerical study has been performed of the transient behaviour of a laminar flow inside a vertical tube submitted to a sudden change of temperature at entry. An implicit method has been used to solve the governing equations of mixed convection. The results allowed the assessment of the influence of a step change in the imposed temperature at entry of the tube and also of the ratio of thermal diffusivities on the velocity and temperature fields.

The existence of a transient instability region was put in evidence, situated in the middle of the tube, between the cold and the hot fluid. The results equally show the axial diffusion in the wall.

\section{Acknowledgments}

The authors are deeply grateful to both the Algerian and the French higher Education Ministries for the BAF grant within the framework of which the present research was done.

\section{References}

[1] Nguyen, C. T., Maïga, S. B., Landry, M., Galanis, N., \& Roy, G., Numerical investigation of flow reversal and instability in mixed laminar vertical tube flow. Int. J. Therm. Sc., 43(8), pp.797-808, 2004.

[2] Morton, B. R., Ingham, D. B., Keen, D. J., \& Heggs, P. J., Recirculating combined convection in laminar pipe flow. ASME. J.Heat Transfer, 111, pp.106-113, 1989.

[3] Bilir, S. \& Ates, A., Transient conjugated heat transfer in thick walled pipes with convective boundary conditions. Int. J. Heat Mass Transfer, 46(14), pp.2701-2709, 2003.

[4] Bernier, M. A. \& Baliga, B. R., Conjugate conduction and laminar mixed convection in vertical pipes for upward flow and uniform wall heat flow. Num. Heat Transfer part A, 21, pp.313-332, 1992.

[5] Bernier, M. A.\& Baliga, B. R., Visualization of mixed convections flows in vertical pipes using a thin semi-transparent gold-film heater and dye injection.. Int. J. Heat Fluid Flow, 13(3), pp.241-249, 1992. 
[6] Zgal, M., Galanis, N., \& Nguyen, C. T., Developing mixed convection with aiding buoyancy in vertical tubes: a numerical investigation of different flow regimes. Int. J. Therm. Sc., 40, pp.816-824, 2001.

[7] Su, Y. C. \& Chung, J. N., Linear stability analysis of mixed convection flow in vertical pipe. J. Fluid Mech., 422, pp.141-166, 2000.

[8] Mai, T. H., Zebiri, R. \& Lorenzo, T., Convection mixte en régime transitoire de couche limite laminaire sur une plaque vertical. C. R. Acad. Sci. Paris, t.329, Série IIb, pp.627-631, 2001.

[9] Mai, T. H. \& Popa, C. V., Numerical study of transient mixed convection in vertical pipe flows. Proc. Of the Conf. On Advances in Fluid Mechanics $I V$, eds. M. Rahman, R. Verhoven \& C. A. Brebbia, WIT Press, pp.75-84, 2002.

[10] Mai, T. H., Popa, C. V. \& Polidori, G., Transient mixed convection flow instabilities in vertical pipe. Heat Mass Transfer, 41, pp.216-225, 2001.

[11] Patankar, S. V., Numerical Heat Transfer and Fluid Flow. Hemisphere, Washington, D. C., 1980

[12] Kakac, S. \& Yener, Y., Convectif Heat Transfer. CRC Press, Boca Raton, 1995. 